\title{
Numerical modeling of 3D discrete breathers in fcc Ni
}

\author{
I.P. Lobzenko ${ }^{1, \dagger}$, P.V. Lobzenko², A.M. Bayazitov ${ }^{3}$, A.P. Chetverikov ${ }^{4}$, \\ R.I. Machmutova ${ }^{5}$, A.A. Kistanov ${ }^{6,7}$ \\ †ivanlobzenko@gmail.com \\ ${ }^{1}$ Institute of Molecule and Crystal Physics, Ufa Scientific Center of RAS, 71 Pr. Oktyabrya St., 450054, Ufa, Russia \\ ${ }^{2}$ North-Caucasian branch of Moscow technical university of communications and informatics, 62 Serafimovicha Street, \\ 344002, Rostov on Don, Russia \\ ${ }^{3}$ Bashkir State University, 32 Z. Validi Street, 450076, Ufa, Russia \\ ${ }^{4}$ Saratov State University, 83 Astrakhanskaya Street, 410012, Saratov, Russia \\ ${ }^{5}$ Bashkir State Agrarian University, 3450 let Oktyabrya Street, 450001, Ufa, Russia \\ ${ }^{6}$ Institute for Metals Superplasticity Problems of RAS, 39 Khalturin Street, 450001 Ufa, Russia \\ ${ }^{7}$ Nanyang Technological University, 50 Nanyang Avenue, 639798 Singapore
}

\begin{abstract}
Molecular dynamics study of discrete breathers in fcc Ni crystal is undertaken. The initial conditions for the excitation of breathers are constructed by imposing a spherically symmetric function, exponentially decreasing with the distance from the center of the sphere, on the delocalized vibrational normal mode, the frequency of which lies above the phonon spectrum of the crystal. This method allows to obtain a three-dimensional discrete breather of a new type, in the core of which the atoms oscillate like in the normal mode, and the amplitude of these oscillations decreases exponentially with distance from the center of the breather. A detailed discussion of the properties of the normal mode on which the breather is built is presented. It is shown that in the two extreme cases, constant volume and zero pressure, the mode shows hard type of non-linearity in a wide range of amplitudes, which ensures the hard type of the nonlinearity of the breather and its frequency lying above the phonon spectrum of the crystal. The spatial localization parameter of the breather is determined as the function of breather amplitude so that the breather life time is maximal. Found discrete breathers have relatively long lifetime of the order of 10 ps, which substantially exceeds the lifetime of thermal fluctuations. The proof of the existence of three-dimensional breathers in fcc $\mathrm{Ni}$, presented in this paper, is an interesting and important result in the theory of discrete breathers.
\end{abstract}

Keywords: discrete breathers, phonons, lattice oscillations, nonlinear oscillations, energy localization, crystal lattice.

\section{Численное моделирование трёхмерных дискретных бризеров в ГЦК решётке $\mathrm{Ni}$}

\author{
Лобзенко И.П. ${ }^{1, \dagger}$, Лобзенко П.В. ${ }^{2}$, Баязитов А.М. ${ }^{3}$, Четвериков А.П. ${ }^{4}$, \\ Махмутова Р.И. ${ }^{5}$ Кистанов А.А. ${ }^{67}$ \\ †ivanlobzenko@gmail.com
}

\footnotetext{
${ }^{1}$ Институт физики молекул и кристаллов Уфимского научного центра Российской академии наук, пр. Октября 71, 450054, Уфа, Россия

${ }^{2}$ Северо-Кавказский филиал Московского технического университета связи и информатики, ул. Серафимовича, 62 , 344002, г. Ростов-на-Дону, Россия

${ }^{3}$ Башкирский государственный университет, ул. З.Валиди, 32, 450076, Уфа, Россия

${ }^{4}$ Саратовский государственный университет имени Н.Г. Чернышевского, ул. Астраханская, д.83, 410012, Саратов,

Россия

${ }^{5}$ Башкирский государственный аграрный университет, ул. 50 лет Октября 34, 450001, Уфа, Россия

${ }^{6}$ Институт проблем сверхпластичности металлов РАН, ул. Халтурина 39, 450001, Уфа, Россия

${ }^{7}$ Наньянский Технологический Университет, 50 пр. Найен, 639798, Сингапур
} 
Методом молекулярной динамики исследуются дискретные бризеры в ГЦК кристалле Ni. Задание начальных условий для возбуждения бризера основано на наложении сферически симметричной, экспоненциально убывающей с удалением от центра сферы функции на делокализованную колебательную нормальную моду, частота которой лежит выше фононного спектра кристалла. Такой метод позволяет получить трёхмерные дискретные бризеры нового типа, в ядре которого колебания атомов повторяют профиль нормальной моды, и амплитуда этих колебаний экспоненциально быстро убывает с удалением от центра бризера. Приведено подробное обсуждение свойств нормальной моды, на основе которой строится бризер. Показано, что в двух предельных случаях, постоянного объёма и нулевого давления, мода демонстрирует жёсткий тип нелинейности в достаточно широком диапазоне амплитуд, что обеспечивает жёсткий тип нелинейности бризера и выход его частоты выше фононного спектра кристалла. Осуществлён поиск параметра пространственной локализации бризера в зависимости от амплитуды колебаний атомов в центре бризера, при котором время жизни бризера оказывается максимальным. Найденные дискретные бризеры имеют сравнительно большое время жизни, порядка 10 пс, что существенно превосходит время существования высокоамплитудных тепловых флуктуаций. До сих пор для чистых металлов был описан лишь один тип дискретных бризеров, а именно, квазиодномерный бризер, локализованный на десятке атомов в одном плотноупакованном атомном ряду. Поэтому доказательство существования трёхмерных бризеров в ГЦК кристалле Ni, представленное в данной работе, является интересным и важным результатом для теории дискретных бризеров, для расширения наших представлений о нелинейной динамике ГЦК решётки, и для возможных приложений концепции дискретных бризеров к объяснению физических процессов, происходящих в ГЦК металлах.

Ключевые слова: молекулярная динамика, нормальная мода, дискретный бризер, ГЦК кристалл.

\section{1. Введение}

Нелинейные колебания в дискретных системах, локализованные в пространстве и периодические во времени, называемые дискретными бризерами (ДБ) [1], представляют большой интерес для различных областей науки [2]. Историческим началом теории ДБ считается работа Сиверса и Такено [4], в которой исследовались локализованные нелинейные моды в модельной цепочке Ферми-Пасты-Улама- $\beta$. С тех пор ДБ были найдены в большом числе физических систем от электрических цепей до Бозе-Эйнштейновских конденсатов. Исследование ДБ проводится как теоретически, так и в реальных экспериментах. На данный момент совершенно очевидно, что ДБ являются характерными объектами для нелинейных дискретных систем.

ДБ в кристаллах являются очень перспективными объектами для изучения, однако их экспериментальное исследование на данном этапе развития науки сопряжено с большими трудностями. В зависимости от степени локализации в бризерном колебании участвуют от единиц до нескольких десятков атомов, что обуславливает возможность их экспериментального обнаружения лишь по косвенным признакам [5-10]. В связи с этим, важную роль при исследовании ДБ в кристаллах играют численные эксперименты. Для определения конкретного вклада бризерных колебаний в такие важные свойства кристаллов как теплоёмкость и теплопроводность, следует сначала подробно изучить свойства ДБ в различных кристаллических структурах.

Методами математического моделирования было уже предсказано существование дискретных бризеров в щелочно-галоидных кристаллах [11 - 13], в металлах $\mathrm{Ni}$ и $\mathrm{Nb}$ [14], V, Fe и W [15], в полупроводниках Si и Ge [16], в упорядоченном сплаве $\mathrm{Pt}^{3} \mathrm{Al}$ [17] и деформированном графене [18-21]. Следует также выделить особую роль первопринципных расчётов при моделировании ДБ [22-24].
Bсе ДБ в чистых металлах, описанные на сегодняшний день, являются квази-одномерными: они локализованы примерно на десятке атомов, расположенных в одном плотноупакованном атомном ряду. Колебания атомов в таком ДБ осуществляются вдоль данного атомного ряда, при этом амплитуда колебаний экспоненциально быстро убывает с расстоянием от центра ДБ [25]. Весь остальной кристалл, фактически, действует как on-site потенциал для данного атомного ряда.

Возникает естественный вопрос, может ли один и тот же кристалл поддерживать ДБ различного типа?

В данной работе дается положительный ответ на данный вопрос. Здесь описаны ДБ, имеющие объемную локализацию колебаний, в отличие от известных квазиодномерных ДБ с колебаниями, локализованными в одном атомном ряду. При этом атомы ядра ДБ обладают смещениями, соответствующими некоторой нормальной моде кристалла. Выбрав нормальную моду, можно сконструировать начальные условия для возбуждения ДБ путём наложения сферически симметричной радиально убывающей функции на данную моду. Максимум этой функции будет соответствовать центру ДБ, смещения атомов будут максимальными в этой области, в то время как при удалении от центра начальные смещения будут плавно спадать. Подробно данный метод задания начальных условий для возбуждения ДБ описан в работе [26].

\section{2. Высокочастотная нормальная мода в $\mathrm{Ni}$}

Дискретный бризер тесно связан с нормальной модой, локализацию на которой мы собираемся ввести, чтобы его построить. Рассмотрим нормальную моду в ГЦК решётке $\mathrm{Ni}$, представленную на Рис. 1. Соответственно паттерну смещений в начальный момент времени, колебания атомов в процессе динамики моды будут приводить к сжатию-растяжению связей атомов, сгруппированных по четвёркам в тетраэдры. По структуре 
смещений атомов можно сделать вывод о том, что данная мода будет обладать высокой частотой по сравнению с другими модами кристалла.

Рассмотрим, как будет меняться частота моды с ростом её амплитуды. В этом случае нам будут интересны два предельных случая: (i) постоянный объём и (ii) нулевое давление в кристалле. При моделировании использовался программный пакет lammps [27] с феноменологическим потенциалом [28]. Для каждого случая была построена зависимость частоты моды от её амплитуды, показанная на Рис. 2. Расчётная ячейка состояла из 8 ячеек трансляции (по две в направлении каждого вектора трансляции) и включала 64 атома.

Видно, что в обоих случаях (постоянного объёма и постоянного давления на границах) нормальная мода обладает жёстким типом нелинейности в достаточно широкой области амплитуд. В силу того, что ядро дискретного бризера полностью повторяет смещения атомов в моде, в процессе колебаний бризер создаёт локальное расширение решётки, аналогичное температурному расширению кристалла. В то же время, вокруг бризера находится невозмущённый кристалл, который сдерживает это расширение.

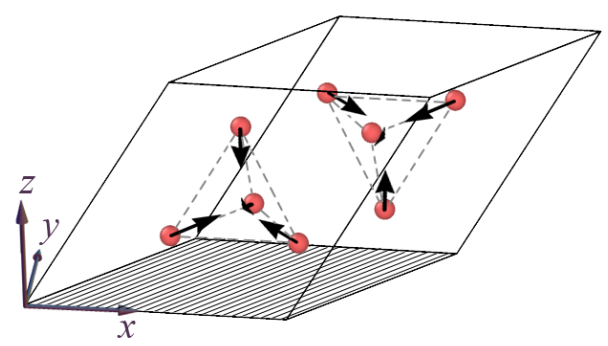

Рис. 1. Движение атомов в рассматриваемой нормальной моде в ГЦК решётке (показана одна трансляционная ячейка). Пунктиром выделены два тетраэдра, в вершинах которых находятся атомы. Атомы совершают колебания к центрам и от центров тетраэдров.

Fig. 1. Normal mode in fcc lattice (one translational cell is shown). Dashed lines show the tetrahedrons with atoms in their vertices. Atoms oscillate toward and backward the center of the tetrahedrons.

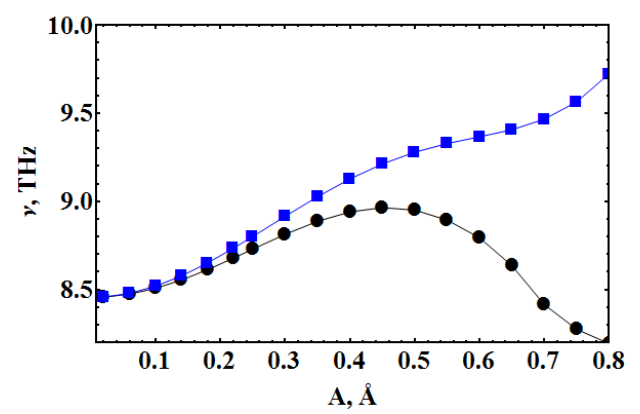

Pис. 2. (Color online) Зависимость частоты нормальной моды, показанной на Рис. 1, от её амплитуды в двух предельных случаях: постоянного объёма расчётной ячейки (синие квадраты) и нулевого давления (чёрные круги).

Fig. 2. (Color online) Dependency of frequency of normal mode, shown in Fig. 1, on its amplitude. Blue squares correspond to the case of constant volume, while black circles correspond to the case of zero pressure.

\section{3. Дискретные бризеры в $\mathrm{Ni}$}

Как уже было сказано выше, профиль смещений ДБ был получен с помощью наложения сферически симметричной радиально убывающей функции на паттерн смещений высокочастотной моды. При этом смещения атомов задавались в следующей форме:

$$
\Delta x_{n, i}^{\alpha}=A \chi_{i}^{\alpha} \frac{1}{\cosh (\beta R)},
$$

где индексы $n, i, \alpha$ обозначают соответственно номер элементарной ячейки, номер атома внутри элементарной ячейки и номер пространственной компоненты смещения атома, $A-$ амплитуда бризера, $\chi-$ паттерн смещений соответствующий нормальной моде, $\beta$ определяет степень локализации бризера, а расстояние $R$ считается от точки, выбранной в качестве центра бризера до данного атома. Поиск бризера для заданной амплитуды сводился к определению оптимального параметра $\beta$, для которого время жизни бризера максимально. Такой подход позволяет получить квазибризеры $[29,30]$ с достаточно большим временем жизни.

На Рис. 3 представлен фрагмент решётки кристалла $\mathrm{Ni}$ со смещениями атомов в начальный момент времени, соответствующими дискретному бризеру. Для данного ДБ амплитуда $A=0.4 \AA$, параметр локализации $\beta=0.345 \AA^{-1}$.

В Табл. 1 приведены данные по полученным ДБ

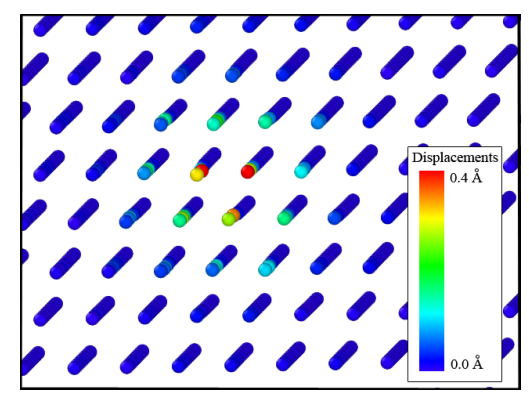

Pис.3. (Color online) Показан срез ГЦК решётки, расположенный так, что видны атомные ряды в направлении (110). Цветом показаны атомы с различными значениями амплитуд колебаний в окрестности ДБ.

Fig. 3. (Color online) Pattern of displacements in the structure of the crystal. A part of the lattice is shown to indicate (110) atomic rows. Color represents atoms with different displacements from their equilibriums according to the color bar.

Табл. 1. Параметры локализации и частоты бризеров различных амплитуд.

Table 1. Localization parameters and frequencies of discrete breathers with different amplitudes.

\begin{tabular}{|c|c|c|}
\hline$A(\AA)$ & $\beta\left(\AA^{-1}\right)$ & $v(\mathrm{THz})$ \\
\hline 0.2 & 0.200 & 8.4644 \\
\hline 0.3 & 0.280 & 8.4725 \\
\hline 0.4 & 0.345 & 8.4788 \\
\hline 0.5 & 0.390 & 8.5021 \\
\hline 0.6 & 0.425 & 8.5207 \\
\hline
\end{tabular}


в ГЦК кристалле $\mathrm{Ni}$. Заметен рост параметра $\beta$, т.е. повышение степени пространственной локализации ДБ, при увеличении его амплитуды. Так же видно, что ДБ обладают жёстким типом нелинейности. Время жизни всех найденных объектов составляет около 100 периодов их колебаний. Так на Рис. 4 изображена зависимость от времени смещения в $y$ направлении атома, являющегося одним из центральных для ДБ. Видно, что около 10 пс данный атом колеблется с существенной амплитудой, что и является временем жизни дискретного бризера.

На Рис. 5 частоты найденных бризеров представлены на фоне частот фононной моды, на которой построен бризер. Как уже было отмечено выше, частоты моды были рассчитаны в двух разных случаях: постоянного объёма кристалла и постоянного (нулевого) давления на его границах. Зависимость частоты от амплитуды ДБ имеет слабый жёсткий характер нелинейности (частота возрастает с ростом амплитуды). В силу того, что найденные бризеры не являются численно точными (частоты разных атомов близки, но не совпадают между собой), амплитудно-частотная характеристика бризера не коррелирует с аналогичной характеристикой моды.

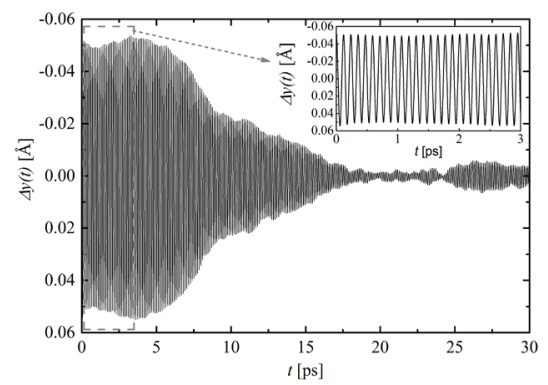

Рис.4. Колебанияцентрального атомадискретного бризеравдоль оси $y$ для случая амплитуды ДБ $A=0.2 \AA$ А̊. Видно, что большая амплитуда сохраняется около 10 пс, что соответствует примерно 100 полным колебаниям центрального атома.

Fig. 4. Oscillations of discrete breather central atom along $y$ axis. The case of $A=0.2 \AA$ is shown. The atom oscillates with high amplitude for about 10 ps, which corresponds to roughly 100 oscillation periods.

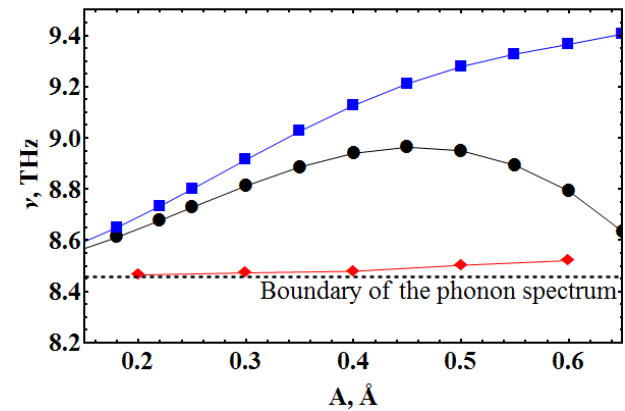

Pис. 5. (Color online) Зависимость частоты нормальной моды, показанной на Рис. 1, от её амплитуды (синие квадраты и чёрные круги), приведённая ранее на Рис. 2, и частота ДБ в зависимости от его амплитуды (красные ромбы).

Fig. 5. (Color online) Frequency of the normal mode shown in Fig. 1 as the function of its amplitude (blue squares and black circles) reproduced from Fig. 2, and frequency of discrete breather as the function of its amplitude (red rhombuses).

\section{4. Выводы}

Методом классической молекулярной динамики исследована высокочастотная нормальная колебательная мода ГЦК решётки Ni. Показано, что при росте амплитуды моды её частота растёт как в случае фиксированного объёма расчётной ячейки, так и при условии термического расширения кристалла в условиях нулевого давления.

С помощью наложения сферически симметричной радиально убывающей функции на смещения атомов в нормальной моде построены дискретные бризеры в ГЦК решётке Ni. Частота дискретного бризера лежит выше фононного спектра кристалла. При увеличении амплитуды бризеров они становятся сильнее локализованными в пространстве и демонстрируют жёсткий тип нелинейности.

Благодарность/Acknowledgements. Работа Л.И. П. и Ч.А. П. выполнена при финансовой поддержке гранта РФФИ № 16-32-50048. Б. А. М. благодарит за поддержку грант Российского научного фонда N 16-12-10175. К. А. А. признателен РФФИ за финансовую поддержку в рамках гранта № 16-32-00479.

\section{Литература/References}

1. S. Flach and A. Gorbach. Phys. Rep. 467, 1 (2007), Doi:http://dx.doi.org/10.1016/j.physrep.2008.05.002

2. D. Campbell, S. Flach and Yu. Kivshar. Phys. Today 51 (1), 43-49 (2004),

3. S.V. Dmitriev, E.A. Korznikova, J.A. Baimova, M. G. Velarde. Physics Uspekhi 186, 446 (2016), DOI: 10.3367/UFNe.2016.02.037729 [C.В. Дмитриев, Е.А. Корзникова, Ю.А. Баимова, М.Г. Веларде. Успехи физических наук 186, 471 (2016), DOI: 10.3367/UFNr.2016.02.037729]

4. A. Sievers and S. Takeno. Phys. Rev. Lett. 61, 970 (1988), Doi:10.1103/PhysRevLett.61.970

5. B. I. Swanson, J. A. Brozik, S. P. Love et al., Phys. Rev. Lett. 82, 3288 (1999), Doi:http://dx.doi.org/10.1103/PhysRev Lett.82.3288

6. G. Kalosakas, A.R. Bishop and A.P. Shreve, Phys. Rev. B 66, 094303 (2002), Doi:http://dx.doi.org/10.1103/PhysR evB.66.094303

7. M.E. Manley, A. Alatas, F. Trouw et al., Phys. Rev. B 77, 214305 (2008), Doi:http://dx.doi.org/10.1103/PhysRevB .77 .214305

8. M.E. Manley, A. J. Sievers, J. W. Lynn et al., Phys. Rev. B 79, 134304 (2009), Doi:http://dx.doi.org/10.1103/PhysR evB.79.134304

9. M. Kempa, P. Ondrejkovic, P. Bourges et al., J. Phys.: Condens. Matter 25, 055403 (2013).

10. A. J. Sievers, M. Sato, J. B. Page and T. Rossler, Phys. Rev. B 88, 104305 (2013), Doi:http://dx.doi.org/10.1103/PhysR evB.88.104305

11. S. A. Kiselev and A. J. Sievers. Phys. Rev. B 55, 5755 (1997), Doi:http://dx.doi.org/10.1103/PhysRevB.55.5755

12. L. Z. Khadeeva and S. V. Dmitriev. Phys. Rev. B 81, 214306 
(2010), Doi:http://dx.doi.org/10.1103/PhysRevB.81.2143 06

13. Yu. A. Baimova, S. V. Dmitriev, A.A. Kistanov, and A. I. Potekaev. Russ. Phys. J. 56 (2), 180 (2013).

14. M. Haas, V. Hizhnyakov, A. Shelkan, M. Klopov, and A.J. Sievers Phys. Rev. B 84, 144303 (2011), Doi:http://dx.doi.org/10.1103/PhysRevB.84.144303

15. R.T. Murzaev, A.A. Kistanov, V.I. Dubinko, D. A. Terentyev, S. V. Dmitriev. Comput. Mater. Sci. 98, 88 (2015).

16. N.K. Voulgarakis, G. Hadjisavvas, P.C. Kelires, and G.P. Tsironis. Phys. Rev. B 69, 113201 (2004), Doi:http://dx.doi.org/10.1103/PhysRevB.69.113201

17. N.N. Medvedev, M.D. Starostenkov and M.E. Manley. J. Appl. Phys. 114, 213506 (2013), Doi:http://dx.doi.org/10.1063/1.4837598

18. L.Z. Khadeeva, S. V. Dmitriev, and Yu. S. Kivshar'. JETP Lett. 97 (7), 539 (2011), Doi: 10.1134/S0021364011190106

19. E. A. Korznikova, J.A. Baimova and S.V. Dmitriev. Europhys. Lett. 102, 60004 (2013).

20. J. A. Baimova, S. V. Dmitriev and K. Zhou. Europhys. Lett. 100, 36005 (2012).

21. B. Liu, J. A. Baimova, S. V. Dmitriev et al., J. Phys. D: Appl. Phys. 46, 305302 (2013).
22. G.M. Chechin, S.V. Dmitriev, I.P. Lobzenko and D. S. Ryabov, Phys. Rev. B 90, 045432 (2014), Doi:10.1103 /PhysRevB.90.045432

23. I.P. Lobzenko, G.M. Chechin, G.S. Bezuglova, Yu. A.Baimova,E. A.Korznikova,S. V.Dmitriev.Phys.Solid State. 58(3), 633 (2016), Doi:10.1134/S1063783416030203

24. G. M. Chechin, I. P. Lobzenko Letters on Materials 4 (4), pp. $226-229$ (2014), 10.22226/2410-3535-2014-4-226-2 29

25. S. V. Dmitriev, L.Z. Khadeeva. Phys. Solid State. 53 (7), 1425 - 1430 (2011), Doi:10.1134/S1063783411070079

26. E. A. Korznikova, S. Yu. Fomin, E.G. Soboleva, S. V. Dmitriev, JETP Letters 103 (4), 277 -287 (2006), DOI: $10.1134 /$ S0021364016040081 [Е.А. Корзникова, С. Ю. Фомин, Э. Г. Соболева, С. В. Дмитриев, Письма в ЖЭТФ, 103 (4), 303 - 308 (2016)]

27. S. Plimpton. J Comp. Phys., 117 (1) $1-19$ (1995), Doi:10.1006/jcph.1995.1039

28. S.M. Foiles, Phys Rev B 32, 7685 (1985), Doi:http://dx.doi.org/10.1103/PhysRevB.32.3409

29. G. M. Chechin, G. S. Dzhelauhova and E. A. Mehonoshina, Phys. Rev. E 74, 036608 (2006).

30. G. M. Chechin, G. S. Dzhelauhova, J. Sound Vib. 322, 490 (2009) . 